\title{
El valor agregado de las redes sociales: propuesta metodológica para el análisis del capital social
}

\author{
Alejandro Velázquez ${ }^{1}$ (UAM) - Luís Rey Marín² (UAC)
}

\section{Resumen}

En el presente trabajo se utiliza una variable reticular para estratificar un grupo de actores sociales, lo cual permite exhibir la posición estructural de sus integrantes en base al origen de su capital social. Se utiliza la variable constricción debido a que integra en una sola medida las dos fuentes principales de capital social: cierre e intermediación. Este enfoque, permite generar hipótesis inmediatas sobre los mecanismos subyacentes a la Posición estructural que actualmente posee cada individuo; sobre las razones por las cuales unos individuos son más exitosos que otros (recursos sociales) y, sobre las acciones posibles para generar efectos agregados que generen valor en la red general.

Palabras clave: Capital social, constricción, recursos sociales, intermediación, cierre, posición estructural.

\begin{abstract}
A In this paper we used a network variable for classifying a real group of social actors inside social capital stratums, it let shows the structural position for each integrant in order to social capital source. Since the individual constraint contains the two principal sources of social capital (closeness and brokerage), we used this one for that. We enhancement how we can to use this approach to generate quickly hypothesis to explain followings: actual individual structural position; mechanisms for upgrade individual social resource; and, strategies to create general effects to realizing the hidden value in the network.
\end{abstract}

Keywords: Social capital, constraint, social resource, Brokerage, closeness, structural position.

\section{Antecedentes}

El término Capital Social no sólo se refiere a sentimientos nobles de amistad y compañerismo, sino que aborda una amplia variedad de beneficios que van desde la confianza, reciprocidad e información, hasta la cooperación en comunidad. El Capital Social genera un valor para la gente que está asociada a él, y también, para quienes están en su perímetro ${ }^{3}$. Es un concepto de suma importancia para explicar el efecto de una red sobre la utilidad en mercados diferenciados.

\footnotetext{
${ }^{1}$ Universidad Autónoma del Estado de México - alexvalvarez@hotmail.com

${ }^{2}$ Universidad Autónoma Chapingo - luisreyuach@gmail.com

${ }^{3}$ Putnam, Robert. Bowling Alone. http://www.bowlingalone.com/socialcapital.php3
} 
Las directrices de investigación sobre el efecto del capital social en el desempeño de una red son múltiples, no obstante, convergen en una premisa: "la estructura social es un tipo de capital que, para ciertos individuos o grupos, puede crear una ventaja competitiva para alcanzar sus fines". Las personas mejor conectadas en una estructura social obtienen mayores beneficios personales, esto implica suponer que las personas exitosas lo son porque de una u otra forma se encuentran mejor posicionadas dentro de una estructura de intercambio social.

En la actualidad, las tendencias de investigación sobre el Capital Social son dirigidas hacia el efecto agregado que éste representa en la red, se busca ante todo descubrir las estructuras sociales y/o normas de conducta que puedan incidir positivamente sobre acciones colectivas ${ }^{4}$.

La presente investigación está dirigida básicamente por la idea de que el capital social individual es un activo importante tanto para describir adecuadamente los mecanismos bajo los cuales se podría incidir positivamente sobre la estructura social general como para mejorar el proceso de aprendizaje y adopción de innovaciones.

De esta manera, el objetivo principal es aportar evidencia que muestre la eficacia de algunos indicadores del análisis de redes sociales para medir: el capital social individual en base a su origen; la posición estructural de los actores de acuerdo al capital social que poseen; y la oportunidad de algunos nodos para intermediar eficientemente las fuentes de información e innovación que consoliden su efecto.

\section{Enfoques reticulares para medir el capital social}

Dos conceptos que serán fundamentales en este trabajo son: capital social de cierre y capital social de intermediación.

De acuerdo con Burt, existen dos formas de medir el capital social desde la perspectiva del análisis de redes sociales: el cierre de la red y los agujeros estructurales.

\footnotetext{
${ }^{4}$ El Banco Mundial se refiere al capital social como "las normas y redes que hacen posible una acción colectiva. Las investigaciones sobre cohesión social -capital social- sugieren que ésta es crítica para aliviar la pobreza y lograr un desarrollo humano y económico sustentable"
} 
Cierre de la red: el efecto asociado con la disminución del riesgo de información incompleta (poco veraz) y por consiguiente asociado a los niveles de confianza existentes entre los integrantes de una red $^{5}$.

Agujeros estructurales: el capital social inherente a los beneficios del control de la información, mismo que produce oportunidades de intermediación (brokerage) a través de los grupos de otra forma desvinculados.

\section{Cierre de la red}

Una red cerrada normalmente asociada con altas densidades, es aquella en la cual la posibilidad de que los individuos que la integran posean la misma información, es elevada (vínculos redundantes, fuertemente cohesionados). Es una red en la cual todos están conectados de tal forma que no es posible que la conducta individual pase inadvertida a los otros, lo cual en términos prácticos involucra a una red densa.

Burt, basado en el argumento de Coleman, plantea que el cierre de la red tiene dos efectos sobre los individuos que la integran:

Primero. Afecta el acceso a la información ya que reduce los costos de intermediación...

Segundo. Facilita sanciones que hacen menos riesgoso para las personas dentro de la red, confiar unas en otras...

Así pues, el cierre de la red plantea que los individuos que pertenecen a una red densa obtienen beneficios directos tanto en la rapidez y veracidad del flujo de información como en los altos niveles de confianza entre los individuos que participan en ella. Esto, por definición propia, es una fuente de capital social.

\section{Agujeros estructurales}

Por otra parte, tenemos como fuente de capital social a las redes de baja densidad. La conexión débil de una red son los agujeros en la estructura social de interacción ${ }^{6}$.

\footnotetext{
${ }^{5}$ Véase (Coleman, 1988).

${ }^{6}$ Véase (Granovetter, 1973) y (Burt, 2004)
} 
Estos agujeros en la estructura social crean ventajas competitivas para un individuo cuya influencia en las relaciones alcanzan a cubrirlos; "Intermediar a través de los agujeros estructurales significa capital social [...] la participación en la difusión de información y el control sobre ella subyacen al capital social de los agujeros estructurales"7 lo cual representa un activo por definición.

La idea de que existan agujeros en la estructura de interacción social en redes de individuos representa simplemente el desinterés mutuo entre los actores sobre sus actividades y su formación es natural en toda red social. El concepto representa una especie de introspección estructural que aísla los flujos de información de los individuos ubicados en lados opuestos del agujero. De esta manera, los nodos que los aprovechen, tienen la oportunidad de intermediar en el flujo de información; generando oportunidades de control sobre la información que poseen los actores anidados en cada extremo, conexión que sin su participación no existiría, esto, por definición es, para aquellos que sepan sacar ventaja, capital social.

\section{Flujos de información en redes abiertas (actores con información incompleta)}

Para ejemplificar de una manera sencilla lo anterior, la Figura 2 muestra el proceso del flujo de la información con respecto al costo de intermediación en grupos con agujeros estructurales y los beneficios de aquellos actores que "aprovechan" entre estos grupos.

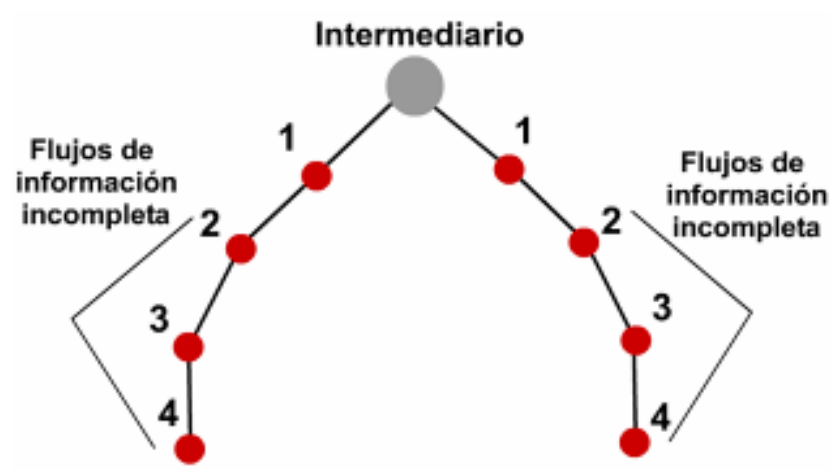

Figura 2. Flujos de información y agujeros estructurales

Se puede observar que existen dos nodos que poseen los más bajos costos de intermediación $($ Costo $=1$ ) gracias a su vínculo directo con el informante (punto de color gris), que en este caso se ha convertido en el principal intermediario de la información fluyente entre los demás nodos, el mismo que se encuentra

\footnotetext{
${ }^{7}$ Véase (Burt, 1992).
} 
aprovechando la conexión en puente, el agujero estructural. Es fácil ver que sin el nodo gris, estos actores quedarían reducidos a dos grupos desvinculados uno del otro.

Por otra parte, podemos observar que fuera de los nodos que tienen vínculos directos $(\operatorname{Costo}=1)$ con el informante, a medida que los demás actores se van alejando del intermediario, sus costos de intermediación se incrementan.

\section{Medidas reticulares utilizadas para evaluar el capital social Densidad}

Es el primer indicador del análisis de redes sociales utilizado en este estudio, este nos permite medir hasta que punto una red se encuentra conectada.

De acuerdo con Burt, bajas densidades indican la presencia de agujeros estructurales mientras que densidades altas implican redes cerradas, las dos fuentes de capital social.

La densidad de la red se mide de una manera simple: Sea $A=\left\{a_{i j}\right\}$ la matriz de adyacencia que caracteriza a la red social de directivos de la red de estudio y $a_{i j}$ la componente relacional que define el vínculo entre el director $\mathbf{i}$ y el director $\mathbf{j}$, tal que, si el actor $\mathbf{i}$ dice tener vínculo con el actor $\mathbf{j}, a_{i j}$ es igual a 1.

Entonces, la densidad estandarizada de la red está dada por la siguiente ecuación:

$$
D=\frac{1}{n(n-1)} \sum_{i=1}^{n} \sum_{j=1}^{n} a_{i j}
$$

Donde:

\footnotetext{
$a_{i j}$ : es la componente relacional entre el director $\mathbf{i}$ y el director $\mathbf{j}$.

$n:$ es el número de actores que integran la red.
}

Es importante mencionar que la forma de medir el capital social de los actores a través de la constricción dependerá de la densidad de la red, ya que como lo hemos mencionado, los dos enfoques para medir el capital social son completamente diferentes.

\section{Cliques}


Esta herramienta nos permite encontrar a aquellos subgrupos cohesionados dentro de la red, el concepto cliqué desde el punto de vista del análisis de redes sociales significa la máxima subred completa posible ${ }^{8}$, un cliqué es, por tanto, un subconjunto de individuos en el que todos los pares de puntos están conectados directamente a través de al menos un vínculo. El tamaño del cliqué se define por el número de actores que lo integran.

\section{Constricción de la red}

En términos reticulares, la Constricción de la red mide hasta que punto una red esta concentrada en contactos redundantes. Esta es una de varias medidas de concentración que pueden usarse con el mismo fin. La constricción de un individuo es alta si los contactos de una persona están directamente vinculados entre ellos (redes densas) o baja cuando los contactos de este se dan indirectamente a través de un contacto central (redes jerárquicas).

\section{Constricción}

La constricción de la red comienza con una medida de cuanto la red de una persona i esta directa o indirectamente dedicada a la relación con el contacto j.

$$
c_{i j}=\left(p_{i j}+\sum_{q=1}^{n} p_{i q} p_{q j}\right)^{2} \quad \forall q \neq i, j
$$

Donde

$$
\begin{aligned}
p_{i j}: \begin{array}{l}
\text { es la proporción tiempo y energía que la red de } \mathbf{i} \text { utiliza para contactar } \\
\text { a } \mathbf{j} .
\end{array} \\
\left(p_{i j}+\sum_{q=1}^{n} p_{i q} p_{q j}\right): \begin{array}{l}
\text { Es la proporción de las relaciones de } \mathbf{i} \text { que están directa o } \\
\text { indirectamente invertidas en la conexión con el contacto } \mathbf{j} .
\end{array}
\end{aligned}
$$

Y donde además se cumple

$$
p_{i j}=\frac{Z_{i j}}{\sum_{q} Z_{i q}}
$$

Donde

\footnotetext{
${ }^{8}$ Véase (Luce y Perry 1949), (Anderson, 1970).
} 
$Z_{i j}$ : $\quad$ representa la fuerza de la relación entre $\mathbf{i}$ y $\mathbf{j}$.

El índice de constricción de la red está dado por la suma de las proporciones cuadradas.

$$
C=\sum_{j=1}^{n} C_{i j}
$$

Esta medida ofrece una prueba sintética que contiene los dos principales mecanismos que proporcionan capital social.

\section{Capital Social vs. Constricción I ndividual}

La investigación presentada por Burt ${ }^{9}$ sobre la medida sintética del capital social, sugiere que la relación entre el origen del capital social de un individuo ( Csi ) y el índice de constricción individual ( $\mathrm{Ci}$ ) es del tipo no lineal.

De manera funcional, este comportamiento se podría representar como un polinomio de segundo grado (una parábola):

$$
C s i=a+b * C i+c * C i^{2}
$$

Gráficamente dicha relación debiera mostrar el comportamiento descrito en la Figura 3.

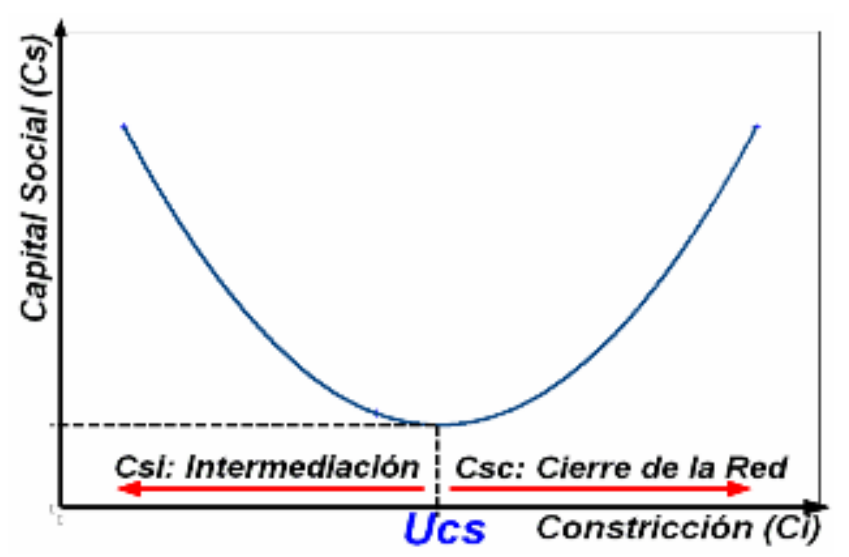

Figura 3. Capital Social vs. Constricción Individual

Donde:

${ }^{9}$ Véase (Burt, 1992). 
Cs: Capital social del individuo "i"

Ci: Índice de constricción del individuo “i”

Ucs: Umbral de acumulación de capital social

Csi: Capital social de intermediación

Csc: Capital social de cierre

Del anterior esquema, es fácil ver que la dinámica que puede seguir la acumulación de capital social de una persona queda completada con los siguientes dos escenarios.

\section{Escenario 1}

$C \mathrm{i}<U C S$ : Entonces podemos decir que el capital social del individuo "i" depende de la intermediación.

\section{Escenario 2}

$C i>U C S:$ Decimos entonces que el capital social del individuo "i" depende del cierre de la red.

Lo subyacente al esquema anterior es que dada una red de $\mathrm{N}$ individuos, los actores que poseen los más bajos niveles de constricción deben el origen de su capital social a la intermediación de agujeros estructurales; y los actores con los niveles más altos deben su capital social al cierre de la red.

Ya que hemos revisado las medidas utilizadas dentro de este artículo, procederemos a comprender la manera de definir el capital social, de acuerdo a las características de la red objeto de estudio.

\section{Caracterización del objeto de estudio}

Analizamos los vínculos de amistad e intercambio de información entre 45 directores de un grupo de empresas dedicadas al sector agropecuario constituidas como asociaciones, regidas todas por un corporativo.

A través de una encuesta generadora de nombres, se visitó a cada uno de los directores con dos preguntas: ¿Con qué otros directores del corporativo mantiene vínculos de intercambio de información referente a la actividad?, ¿Quiénes son los directores del corporativo con los que mantiene una relación de amistad más allá de los deberes laborales? 
Gracias al apoyo del corporativo, las respuestas obtenidas de estas dos preguntas, más algunos atributos de cada director y de la empresa que dirige (edad, años de experiencia en el puesto, número de clientes en la empresa, etc.), permitieron armar una base de datos consistente para el análisis.

\section{Densidad: el sentido funcional de la constricción}

Basándonos en un primer nivel de análisis con respecto a los datos reticulares obtenidos en el trabajo de campo, se puede observar a simple vista en la Figura 4, que la mayoría de los Directores están vinculados entre sí, no obstante, la red presenta una densidad general de tan sólo $6.4 \%$, lo que representa, en términos prácticos, una red de baja densidad donde se espera encontrar la presencia de agujeros estructurales, una de las fuentes de capital social.

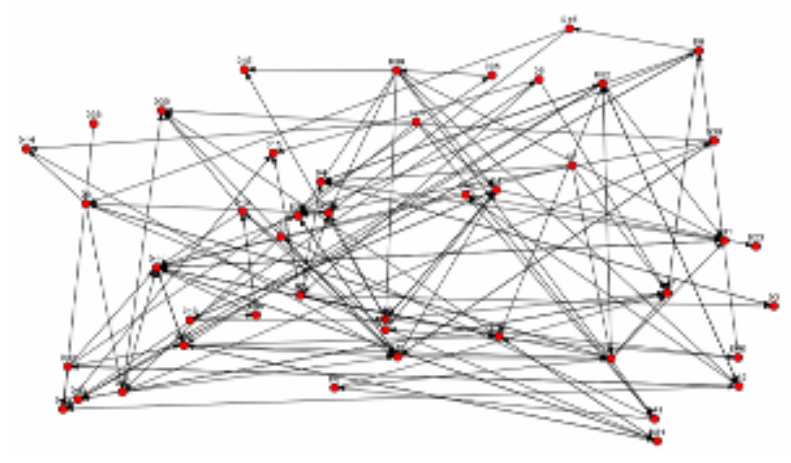

Figura 4. Red de directores.

Gracias al primer nivel de análisis, se espera que la red exhiba contraste entre grupos cerrados y grupos abiertos, por ello, procedimos a buscar a aquellos nodos que posean vínculos redundantes para tener un marco de comparación entre subredes cerradas y subredes con agujeros estructurales dentro de nuestra red a través de un análisis de cohesión utilizando la herramienta n-cliqué ${ }^{10}$.

Por lo que el segundo nivel de análisis consistió en encontrar al máximo número de miembros pertenecientes a un cliqué en la red. El cliqué más alto fue de cuatro individuos.

\footnotetext{
${ }^{10}$ Véase (Alba, 1973) y (Bock; y Husain; 1950).
} 


\section{Análisis de cohesión - Ncliqué, Vínculos redundantes}

En nuestra red encontramos que únicamente existían dos subredes completamente cerradas (nodos fuertemente vinculados entre ellos) de cuatro individuos cada una, véase figura 5 .

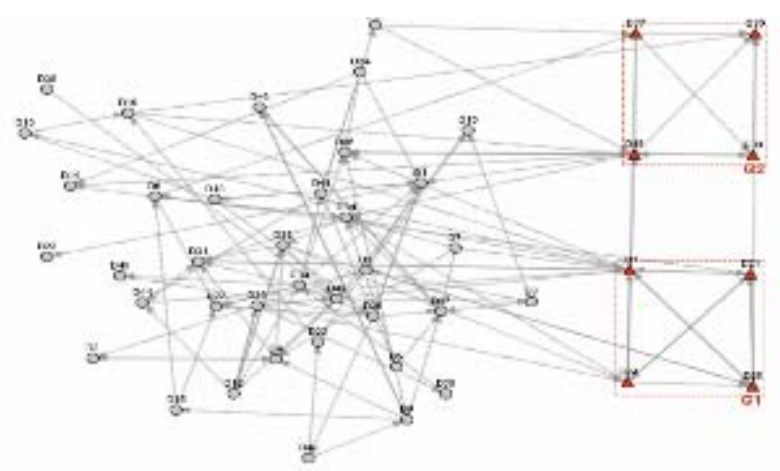

Figura 5. Subredes de la red de directores.

En estos subgrupos (actores con información completa), podemos esperar que la conducta que los rige obedezca a altos niveles de confianza, pues los costos sociales de algún cambio repentino de conducta, al interior del cliqué, son elevados.

De esta manera, los vínculos redundantes, permiten que todos los actores posean la misma importancia, por lo que el flujo de información es constante y completo. El capital social al interior de estos grupos, depende del cierre de la red $^{11}$.

Por otro lado, el análisis de cohesión arroja a un tercer subgrupo de actores los cuales no pertenecen a grupos cohesionados de cuatro individuos. A este conjunto le llamaremos "actores con información incompleta".

Dentro de la subred de "actores con información incompleta" las oportunidades de intermediación son elevadas ya que el control de la información subyace en pocos actores de la red permitiendo el control de la misma y la tendencia a la existencia de información incompleta (susceptible de ser controlada).

${ }^{11}$ Véase (Coleman, 1988). 


\section{El capital social de las subredes cerradas. Sobre los grupos cohesionados, sus miembros y su posición estructural en la red}

Retomando el análisis de cohesión antes realizado, recordemos que tenemos a sólo dos cliques de cuatro individuos cada uno.

La gran mayoría de los actores inmersos en estos cliques (Véase Figura 5), poseen, por derecho propio, una posición estructural importante por el simple hecho de pertenecer a los grupos bien cohesionados. Esta situación, apoyada con lo mencionado en párrafos anteriores sobre los efectos del cierre de la red, crea la posibilidad de institucionalizar reglas informales de conducta basadas en confianza, reciprocidad, información y cooperación.

Para el caso particular de nuestro objeto de estudio, mencionaremos dos ejemplos donde se puede observar lo anterior.

Contexto: se realizó una reunión participativa con los directores con la finalidad de hacer una propuesta federal de subsidio a gran escala en beneficio del corporativo. Después de una breve exposición por parte de los organizadores se sucedió la participación interactiva entre organizadores y directores para acordar normas y estrategias de acción congruentes con este objetivo. Se observó el comportamiento y participación de cada director tomando registro de ello a través de una bitácora donde el encargado de llevarla a cabo se encontraba ubicado de manera discreta en la reunión. Se observaron los siguientes hechos.

Hecho1 Los directores que mayor conocimiento e interés mostraron, fueron en : $\quad$ su totalidad los pertenecientes a los dos grupos cohesionados dentro de la red, mientras que sólo unos cuantos individuos de los que no pertenecían a esos grupos mostraron casi nulo interés y conocimiento sobre el tema.

Hecho2 Los directores pertenecientes a los grupos bien cohesionados : mostraron mayor disponibilidad para realizar negocios ${ }^{12}$ en conjunto (y cooperando con el resto de los directivos) para el beneficio de las empresas que dirigen, siempre y cuando fueran con los miembros de su mismo grupo, mientras que sólo unos cuantos individuos que no pertenecían a esos grupos mostraron tal disponibilidad

Finalmente, estos ejemplos nos sugieren que el argumento de Burt y Coleman sobre los efectos del cierre de la red en los individuos no distan de la realidad.

\footnotetext{
12 Tales como: compra de insumos en conjunto: adquiriéndolos a un menor costo, con distribución de los mismos de acuerdo al capital proporcional correspondiente a la inversión y sin contrato previo.
} 
Ahora, de acuerdo al cierre de la red, se calculó la constricción individual de los actores de ambos grupos, los cuales se presentan de la siguiente manera:

\begin{tabular}{ccc}
\hline Actor & Grupo & Constricción agregada individual \\
D11 & 1 & 0.882 \\
D21 & 1 & 0.882 \\
D28 & 1 & 0.970 \\
D29 & 1 & 0.970 \\
\hline D18 & 2 & 0.865 \\
D30 & 2 & 0.865 \\
D36 & 2 & 0.997 \\
D37 & 2 & 0.997 \\
\hline
\end{tabular}

Cuadro 1. Índice de constricción agregada individual por cliqué. Subredes cerradas.

Para el caso específico de los miembros de los cliques (tal como lo muestra el cuadro anterior) al hablar de subredes cerradas (cohesionadas - alta densidad) la relación entre la constricción y el capital social se lee de manera directa, debido a que el capital social en este caso esta dado por el cierre de la red.

De esta manera, el cuadro anterior nos muestra que los actores de más alto índice de constricción son aquellos que poseen el mayor capital social de cierre.

En la Figura 6.a se presenta la posición estructural de los 8 actores pertenecientes a las dos subredes cerradas (nodos de forma triangular) en la red, cuya estructura individual se presenta en la Figura 6.b.

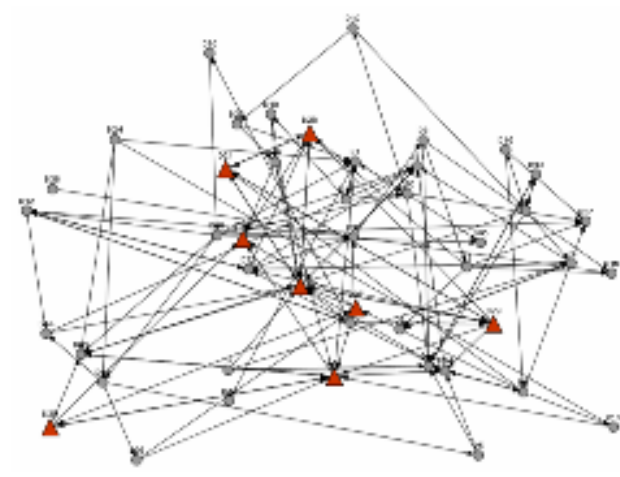

Figura 6.a

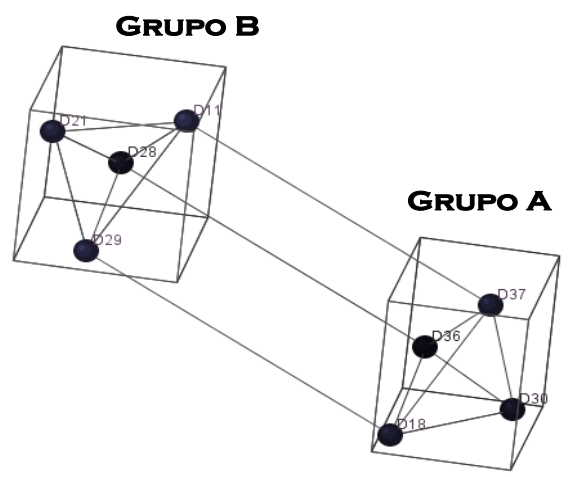

Figura 6.b

Figura 6. Representación de los actores pertenecientes a las dos subredes 


\section{El capital social de las redes abiertas: la constricción individual de los actores y su interpretación}

Una vez revisadas las subredes cohesionadas, el capital social de cierre, la posición estructural de sus miembros y la manera de interpretar la constricción, procederemos a calcular el capital social de todos los nodos de la red.

De acuerdo con nuestro planteamiento, ubicamos que dada la baja densidad general de nuestra red $(6.4 \%)$ el origen del capital social que posee cada individuo se encuentra interpretando la constricción de la manera siguiente: "a mayor constricción del director, el capital social que éste posee se lo debe a que su egored es cerrada y entre más baja sea su constricción su capital social se lo debe en mayor medida a la intermediación de agujeros estructurales".

Por lo que de los 45 directores, aquellos que cuenten con los más bajos niveles de constricción el origen del capital social que éstos poseen se lo deben a la intermediación; y los niveles más altos de constricción deben el origen de su capital social al cierre.

Lo anterior nos permitirá encontrar a los individuos que poseen el mayor capital social de intermediación ( $C s i$ ), actores a través de los cuales fluye la mayor parte de la misma.

\begin{tabular}{cccccc}
\hline Actor & $\begin{array}{c}\text { Constricción } \\
\text { Agregada } \\
\text { Individual }\end{array}$ & Actor & $\begin{array}{c}\text { Constricción } \\
\text { Agregada } \\
\text { Individual }\end{array}$ & Actor & $\begin{array}{c}\text { Constricción } \\
\text { Agregada } \\
\text { Individual }\end{array}$ \\
\hline D1 & 0.215 & $\mathbf{D 1 6}$ & 0.545 & $\mathbf{D 3 1}$ & 0.248 \\
\cline { 2 - 6 } D2 & 0.271 & $\mathbf{D 1 7}$ & 0.252 & $\mathbf{D 3 2}$ & 0.211 \\
\cline { 2 - 6 } D3 & 0.705 & $\mathbf{D 1 8}$ & 0.187 & $\mathbf{D 3 3}$ & 0.281 \\
\cline { 2 - 6 } D4 & 0.246 & $\mathbf{D 1 9}$ & 0.261 & $\mathbf{D 3 4}$ & 0.184 \\
\cline { 2 - 6 } D5 & 0.386 & $\mathbf{D 2 0}$ & 0.267 & $\mathbf{D 3 5}$ & 0.500 \\
\cline { 2 - 6 } D6 & 0.209 & $\mathbf{D 2 1}$ & 0.450 & $\mathbf{D 3 6}$ & 0.349 \\
nyyyyy D7 & 0.302 & $\mathbf{D 2 2}$ & 0.333 & $\mathbf{D 3 7}$ & 0.290 \\
n18 & 0.396 & $\mathbf{D 2 3}$ & 1.000 & $\mathbf{D 3 8}$ & 1.000 \\
\cline { 2 - 6 } D9 & 0.267 & $\mathbf{D 2 4}$ & 0.250 & $\mathbf{D 3 9}$ & 0.158 \\
\cline { 2 - 6 } D10 & 0.397 & $\mathbf{D 2 5}$ & 0.393 & $\mathbf{D 4 0}$ & 0.333 \\
\cline { 2 - 6 } D11 & 0.173 & $\mathbf{D 2 6}$ & 0.235 & $\mathbf{D 4 1}$ & 0.200 \\
\cline { 2 - 6 } D12 & 0.284 & $\mathbf{D 2 7}$ & 0.263 & $\mathbf{D 4 2}$ & 0.282 \\
\cline { 2 - 6 } D13 & 0.509 & $\mathbf{D 2 8}$ & 0.327 & $\mathbf{D 4 3}$ & 0.643 \\
\cline { 2 - 6 } D14 & 0.333 & $\mathbf{D 2 9}$ & 0.313 & $\mathbf{D 4 4}$ & 0.307 \\
\cline { 2 - 6 } D15 & 0.416 & $\mathbf{D 3 0}$ & 0.498 & $\mathbf{D 4 5}$ & 0.625 \\
\hline
\end{tabular}

Cuadro 2. Índice de constricción agregada individual. 
Los índices de constricción individual para la red de Directores se reportan en el Cuadro 2.

\section{Estratos de constricción}

Después de calcular la constricción individual, procedimos a sistematizar la información con dos pasos: Primero, se organizaron los resultados de tal manera que se pudiesen ubicar a los actores con más bajo nivel de constricción -los poseedores del mayor capital de intermediación-, y se les asignó un atributo que los estratificara de acuerdo a su índice de constricción, de esta manera, se obtuvieron cinco estratos (detallados en el Cuadro 3), los cuales nos permitirán precisar de manera simple a aquellos actores mejor posicionados en la red.

\begin{tabular}{llcc}
\hline \multicolumn{1}{c}{ Estrato } & \multicolumn{1}{c}{ Rango de clase } & Cantidad & \% \\
\hline Estrato I & Menor de 0.25 & 11 & 24.4 \\
\hline Estrato II & de $0.25-0.34$ & 19 & 42.2 \\
\hline Estrato III & de $0.35-0.44$ & 5 & 11.1 \\
\hline Estrato IV & de $0.45-0.54$ & 5 & 11.1 \\
\hline Estrato V & de $0.55-1$ & 5 & 11.1 \\
\hline Total & & 45 & 100 \\
\hline
\end{tabular}

Cuadro 3. Estratificación de los Directores de acuerdo a su índice de constricción.

El Estrato I nos ubica a los actores poseedores del más elevado capital social de intermediación en la red, mientras que el Estrato II contiene a los actores que poseen el segundo nivel de capital y así sucesivamente hasta el Estrato V, referente a los individuos de menor capital social de intermediación.

\section{La posición estructural de los individuos y la intermediación de la información de acuerdo al índice de constricción individual}

En la Figura 7 mostramos el patrón de interacciones de la red general por estrato de constricción, las Figuras 7.a y 7.d muestran a la red completa; de estas dos figuras parten algunos ejercicios: la Figura 7.b muestra a la red sin el Estrato I, podemos observar que el número de vínculos es mayor que en la Figura 7.e la cual muestra el número de vínculos sin el Estrato II. Esta diferencia en el número de vínculos nos hace reflexionar sobre el papel que juegan los actores de acuerdo al Estrato relacional al que pertenecen, parece ser, que los Estratos III, IV y V dependen un poco más de aquellos actores del Estrato II para vincularse a la red, no obstante, de acuerdo a la Figura 7.h, el Estrato II por si solo, muestra ciertas deficiencias en su patrón de interacciones ya que claramente se observan vínculos no redundantes por lo que la presencia de agujeros estructurales es obvia. 


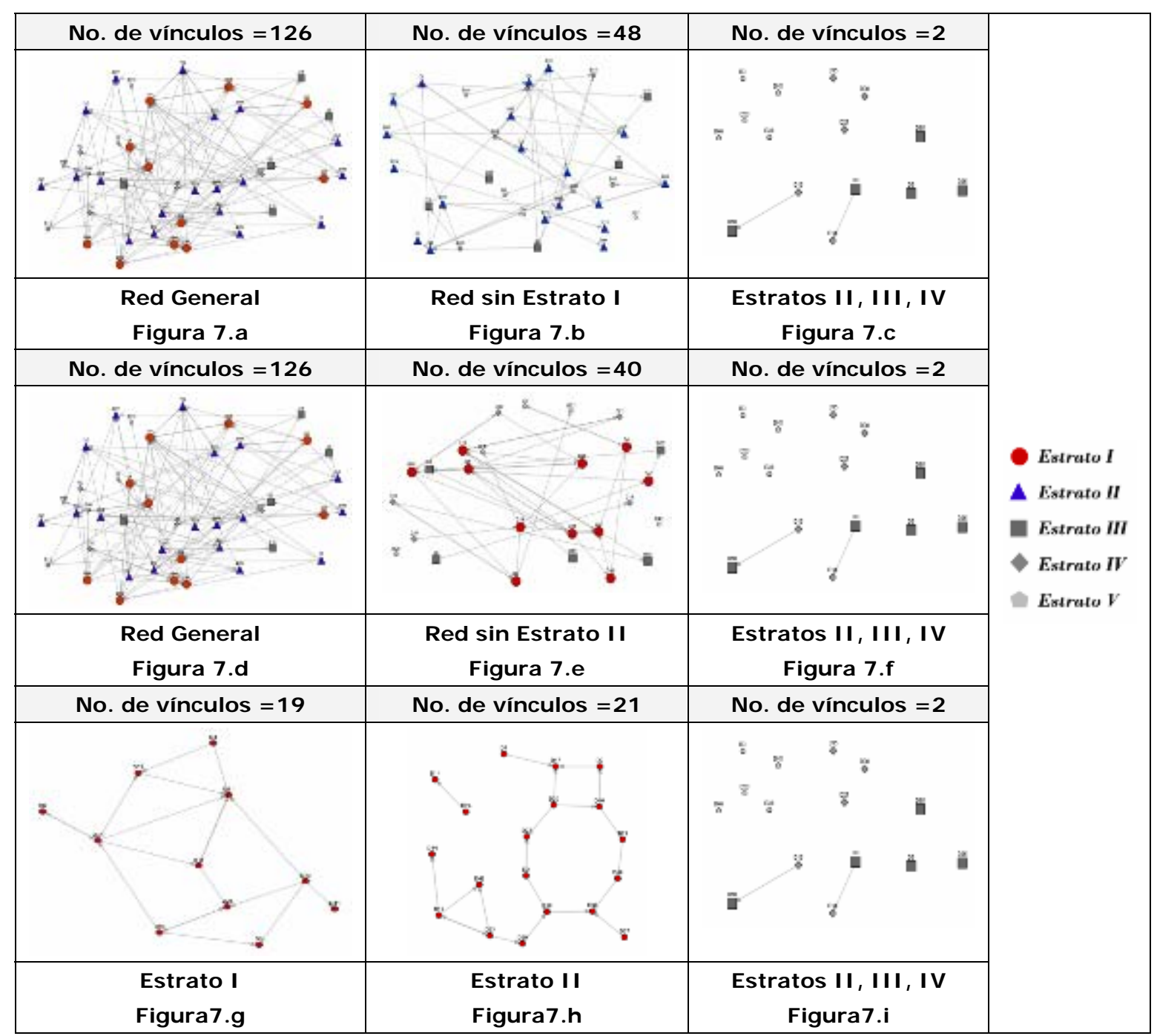

Figura 7. Patrón de interacciones de la red general por estrato de constricción.

De esta manera, podemos deducir que la intermediación de la información corre de manera escalonada, es decir, el Estrato II aprovecha los agujeros estructurales de los Estratos III, IV y V mientras que el Estrato I hace lo propio con el estrato II.

Dicho lo anterior, surgen entonces un par de preguntas por demás interesantes: ¿porqué la intermediación de la información sigue un comportamiento escalonado de acuerdo a la constricción?, ¿cuál es la constricción individual de aquellos actores pertenecientes a los grupos cohesionados en la red? 
Aquellos actores pertenecientes a las subredes cerradas poseían por ese simple hecho, una posición estructural importante, sin embargo, no todos ellos poseen capital social de intermediación alto. En la Figura 8 se muestran los Estratos de aquellos individuos pertenecientes a los dos cliques encontrados en nuestro estudio.

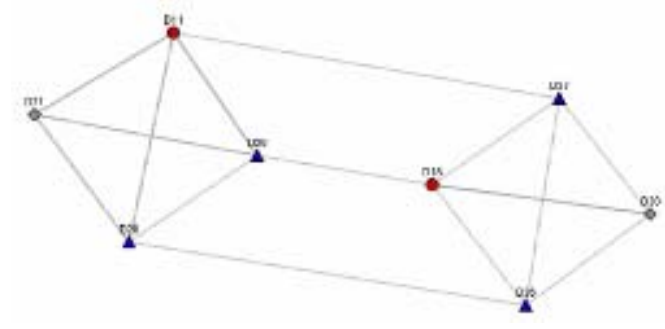

Figura 8. Estratos de los dos cliques

La Figura anterior nos muestra un comportamiento coincidentemente muy similar en cuanto al ranking de los nodos respecto a la constricción individual, podemos observar que los dos subgrupos poseen el mismo tipo de actores, tenemos que los nodos de color gris en las dos subredes (Estrato IV) se encuentran vinculados con dos actores del Estrato II y un solo actor del Estrato I. Esto, de una u otra forma nos indica que las subredes poseen el mismo comportamiento de la red general ya que a simple vista la Figura 8 muestra un escalonamiento muy similar al presentado en la Figura 7.

Pero, ¿por qué la intermediación de la información sigue un comportamiento escalonado de acuerdo a la constricción? Esto quizá se deba a una serie de factores que pueden ir mucho más allá de las simples relaciones de confianza, amistad y comunicación: En términos generales, puede ser muy complejo que los actores que menos capital social poseen tengan vínculos directos con los mejor posicionados, recordemos que los poseedores del capital social poseen un valor agregado que puede resultar difícil de compartir. Quizá la Figura 3 nos pueda ejemplificar mejor lo antes mencionado.

De esta manera, utilizar una variable reticular para estratificar al conjunto de actores de una red, permite visualizar de manera inmediata algunas características de la posición estructural de los actores en la red; misma que permite plantear hipótesis inmediatas que pueden ser esclarecidas utilizando las variables de atributos. 


\section{Características de los Directores. ¿El capital social de los individuos depende de atributos cualitativos?}

Si bien es cierto que el capital social de intermediación permite caracterizar a los actores en función de sus activos sociales individuales, estos, de alguna manera, poseen ciertos atributos tales que de una $u$ otra forma les han ayudado a posicionarse dentro de su estructura social. El Cuadro 4 puede dar cuenta de ello.

\begin{tabular}{lcccc}
\hline Estrato & Años en función & Escolaridad (años) & Edad (años) & $\begin{array}{c}\text { Número de } \\
\text { Socios }\end{array}$ \\
\hline Estrato I & $\mathbf{4 . 8}$ & $\mathbf{1 3 . 9}$ & $\mathbf{4 1 . 8}$ & $\mathbf{1 5 9 1 . 7}$ \\
\hline Estrato II & 8.8 & 9.8 & 58.9 & 1000.7 \\
\hline Estrato III & 3.4 & 12.4 & 46.4 & 533.4 \\
\hline Estrato IV & 8.4 & 12.4 & 44.4 & 1003.4 \\
\hline Estrato V & 7.0 & 10.8 & 48.4 & 767.6 \\
\hline Total & 7.0 & 11.5 & 50.6 & 1067.6 \\
\hline
\end{tabular}

Cuadro 4. Atributos de los directores por Estrato de constricción.

El Cuadro anterior muestra claramente que el Estrato I (poseedor del mayor nivel de capital social de intermediación) posee diversos atributos en promedio que los demás Estratos no presentan: el más alto número de socios, los directores más jóvenes y de más alta escolaridad y algo muy importante, pocos años en función como directores (en comparación con los demás estratos, exceptuando al Estrato III).

Esto último nos revela que algunos de los directores que más tiempo tienen laborando (Estratos IV y V), no tienen tanta preocupación por controlar la información, pues quizá su principal interés no radica en renovar e intermediar los vínculos sociales con sus similares (se enfocan en sus propias actividades).

En contraste, los individuos que tienen el menor tiempo fungiendo el papel de director (Estrato III), con aun muchas expectativas (laborales y profesionales), estarán más interesados en invertir tiempo generando vínculos que en su momento, les permitan la oportunidad de intermediación, la Figura 7.i, muestra claramente que los Directores D10 y D8 mantienen vínculos con los Directores D13 y D16 (estos últimos del estrato IV), debido a la experiencia laboral que estos presentan. 
Esto quizá quiera decir que algunos Directores del Estrato III están intentando posicionarse estructuralmente a través de los directores con experiencia, pero con poco capital social de intermediación, sin contemplar aun, vincularse directamente al Estrato I ya que como lo habíamos mencionado, no es tan fácil llegar de forma directa a los mejor posicionados.

Por otra parte, un caso totalmente diferente presenta el Estrato II, ya que a pesar de ser el grupo de más edad y menor escolaridad en promedio, sus miembros se encuentran estructuralmente bien posicionados, esto quizá, por la cantidad de años de experiencia en el puesto.

Esto nos hace reflexionar sobre el desempeño social de los actores con respecto al tiempo laborando, ya que tenemos dos casos totalmente opuestos: el Estrato I con 4.8 años laborando y el Estrato II con 8.8 años.

Desde este punto de vista, podemos deducir que la experiencia laboral no es un factor determinante para la posesión del capital social de intermediación.

Sin embargo, hay diferencias estructurales que nos arrojan lo contrario: A diferencia de los Estratos IV y V, los miembros del Estrato II quizá supieron, en su momento, aprovechar las oportunidades de intermediación a su alcance, no obstante, al ver su red social (Figura 7.h) se aprecian claramente algunos agujeros estructurales, ya que si observamos detenidamente su red podremos darnos cuenta que sólo el $42 \%$ de los nodos poseen dos vínculos mientras que los demás tienen una sola interacción, esta ineficiencia, dada a través del tiempo, y gracias a los constantes cambios de Directores, fue desarticulando su red, y ésta es actualmente aprovechada por aquellos Directores cuyos vínculos alcanzan a cubrirlos, específicamente, el Estrato I.

Por lo tanto, existe cierta relación entre la experiencia en el puesto y otros atributos con la posesión del capital social de intermediación ya que este no se genera de la nada, los actores que logran la intermediación de la información son normalmente personas con otro tipo de visión gracias a su formación, empuje, oportunidades, y sobretodo, su esfuerzo.

Después de todo, recordemos que el proceso de acumulación de capital social radica en la inversión de esfuerzo dedicado a conseguir conexiones, poseerlo conlleva beneficios pero también costos en tiempo de inversión para la generación de vínculos y control de la información. 


\section{Conclusiones}

Existen dos características del capital social. La primera es que la intermediación de la información es una fuente de valor agregado, sin embargo, la posibilidad de institucionalizar reglas informales de confianza, reciprocidad, información y cooperación (cierre de la red) puede ser crítica para consolidar ese valor.

Como evidencia, tenemos que la información entre los actores pertenecientes a redes cerradas circula de manera fluida y veraz gracias a los vínculos de confianza y a los costos sociales (castigos sociales informales) que estos conllevan. De esta manera, aunque no todos los individuos cohesionados poseen altos niveles de capital social, todos ellos tienen acceso directo al capital social de intermediación gracias a sus vínculos directos con aquellos nodos, pertenecientes a su mismo grupo, que lo controlan.

Por otro lado, tenemos que los actores que poseen el capital social de intermediación se encuentran con la mejor posición dentro de la red, lo cual les permite intermediar una serie de flujos de información que van desde noticias y oportunidades de negocio hasta "chismes" de sus similares. Así, la intermediación a través de los agujeros estructurales es más valiosa para la gente que tiene pocos competidores, para los grupos donde los individuos se encuentran fuertemente vinculados y para los mercados donde las organizaciones están fuertemente interconectadas.

De esta manera, el poseer capital social ya sea de cierre o de intermediación es, por derecho propio, un valor agregado individual al cual no todos pueden acceder ya que la inversión en tiempo para adquirir los contactos "correctos" es costosa, pero muy alentadora. Aquellos que no pueden acceder a ese capital social correrán el riesgo de poseer información de segunda mano y quizá pasada de moda, lo cual los empuja a trabajar para poseer capital social de intermediación a través de "contactos correctos" o de cierre, donde su posición dentro de un grupo cohesionado les permitirá adquirir información crucial para agregarse valor.

La investigación da cuenta de lo siguiente: en una red existen subredes que se encuentran fuertemente interconectadas y subredes de baja densidad, coexistiendo ambas dentro de una misma estructura social, siendo los actores inmersos en ella, afectados simultáneamente por ambos efectos. 
De estas reflexiones surge una pregunta que tal vez, en futuras investigaciones, nos permita encontrar el valor escondido de las redes: ¿es correcto invertir en la formación de grupos fuertemente cohesionados (con capital social de cierre) con niveles de confianza elevados, sin atender los vínculos que articulen a un grupo cerrado con otro (capital social de intermediación)? ¿O se debe buscar algún tipo de equilibrio?

Quizá sólo sean suposiciones, pero seguramente una de las premisas fundamentales del análisis de redes sociales sea encontrar el valor aquel valor escondido que nos permita comprender los mecanismos del flujo de información e innovaciones entre individuos, eficientes para agregar valor a una red.

\section{Bibliografía}

Alba, R.D. (1973). A graph-theoretic definition of a sociometric clique. Journal of Mathematical Sociology 3:113-126.

Anderson, S.S. (1970): Graph Theory. Chicago: Markham Publishing Company.

Bock, R.D., and Husain, S.Z. (1950). An adaptation of Holzinger's B-coefficients for the analysis of sociometric data. Sociometry, 13, 146-153.

Burt, R.S. (1992) Structural holes: The social structure of competition. Cambridge: Harvard University Press.

Burt, R.S., R. Hogarth and C. Michaud, (2000) "The Social Capital of French and American Managers," Organization Science.

Burt, R.S. (2004) Structural holes and Good ideas. American Journal of Sociology 110, 2:350-399.

Burt, R.S. (2005). Brokerage and Closure: An Introduction to Social Capital. Oxford: Oxford University Press.

Coleman, James S. (1988). Social Capital in the Creation of Human Capital. American J ournal of Sociology, 94, (Supplement), S95-S120

Coleman, James S. (1990) Foundations of Social Theory. Cambridge, MA: Harvard University Press.

Granovetter, Mark S. (1973). The Strength of Weak Ties. American Journal of Sociolology 78: 1360-80.

Luce, R. D. y Perry, A. (1949). A method of matrix analysis of group structure. Psychometrika, 14.

Rogers M. Everett. (1962) Diffusion of innovations. The Free Press. New York. 